\title{
APPLICATION OF BICYCLIC ISOTHIOUREA MOIETY FOR THE DESIGN OF NEW ANTICANCER AGENTS
}

\author{
$\underline{\text { A. Alexeev }}{ }^{1}$, A. Evdokimova', I. Elisseev', E. Nurieva', E. Milaeva', ${ }^{1,2}$, \\ S. Kuznetsov ${ }^{3}$ and O. Zefirova ${ }^{1,2}$ \\ ${ }^{1}$ Department of Chemistry, M.V. Lomonosov Moscow State University, \\ 119992 Russia, Moscow, Leninskie gory, 1/3. \\ ${ }^{2}$ Institute of Physiologically Active Compounds, Russian Academy of Sciences, \\ 142432 Russia, Chernogolovka, Severny pr., 1. \\ ${ }^{3}$ Institute of Biological Sciences, University of Rostock, D-18059 Rostock, Germany.
}

DOI: 10.19163/MedChemRussia2021-2021-379

E-mail:Josef393@gmail.com

A vast majority of aromatic and non-aromatic heterocyclic moieties is used in the process of drug design. Cyclic isothioureas are relatively rare applied in this process, though this moiety is present in the structures of some clinically used drugs and drug candidates at various stages of preclinical trials (e.g. Levamizole, Xylazine, Elenbecestat). Literature search reveals examples of successive replacement of aromatic group (thiazole, pyridine etc.) in the lead molecule by cyclic isothiourea moiety [1]. In this work we carried out an analogous replacement in the structure of verubulin, a potent (but not clinically approved) ligand of colchicine binding site of tubulin [2]. Bicyclic isothioureas of $\mathrm{N}$-(4-methoxyphenyl)-2-aminocycloalkane[d]thiazol type were synthesized by intramolecular electrophilic cyclization of $\mathrm{N}$-(cycloalk-2-enyl)-N'-(4methoxyphenyl)thioureas under the conditions described in refs. [6-8]:

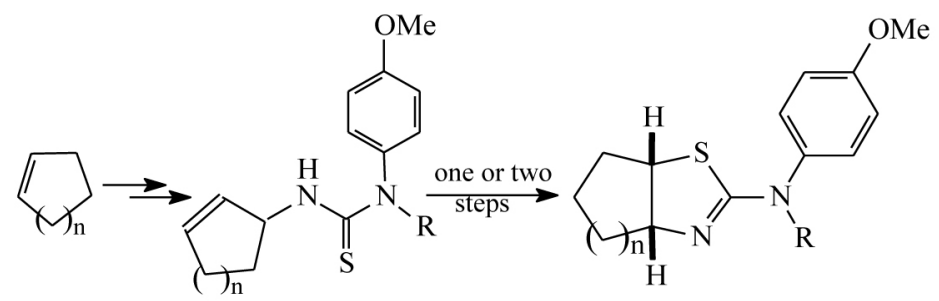

Isothiourea fused with seven-membered ring caused noticeable changes of the morphology of human lung carcinoma cells A549, but without affecting their microtubule net.

The applications of bicyclic isothiourea moiety for the modification of the structures of other tubulin-targeted agents (colchcine, podophyllotixin etc.) was also studied and lead to several active antimitotic agents with some selectivity to cancer cells A549.

The work was supported by Russian Science Foundation (project 19-13-00084).

\section{References}

[1] E. V. Nurieva, A. A. Alexeev, O. N. Zefirova. Cyclic isothiourea in drug design. Chem. Heterocycl. Comp. In press.

[2] N. Sirisoma, A. Pervin, H. Zhang et al. J. Med. Chem., 2009, 52, 2341.

[3] E. V. Nurieva, T. P. Trofimova, A. A. Alexeev et al. Mendeleev Commun., 2018, $28,390$.

[4] A. A. Alexeev, E. V. Nurieva, T. P. Trofimova et al. Mendeleev Commun., 2019, $29,14$.

[5] A. A. Alexeev, E. V. Nurieva, K. A. Lyssenko, Yu. K. Grishin, O. N. Zefirova, Struct. Chem., 2019, 30, 473.

[6] E. V. Nurieva, A. A. Alexeev, Y. K. Grishin, V. A. Tafeenko, O. N. Zefirova. Mendeleev Commun., 2020, 30, 145 . 